\section{A Case study of Group B Streptococcus Associated with Women in Antepartum Period and their Neonates, Ile Ife South-western Nigeria}

\section{Abstract}

Streptococcus agalactiae colonizes the genital and gastrointestinal tract. In pregnancy, vertical transmission of GBS to the new-born can cause neonatal sepsis, pneumonia and meningitis. The aim of this study was to determine the prevalence of GBS in pregnant women in their third trimester, frequency of neonatal colonization and Antibiotics Susceptibility of the isolates recovered at the Obafemi Awolowo University Teaching Hospital Complex (OAUTHC) IleIfe, Osun State, Nigeria. Rectum and vaginal swabs were collected from a total number of 24 third trimester pregnant women between 35 - 37 weeks of gestation and their neonate delivered at OAUTHC both at labour ward and labour ward theatre. The samples were cultured in Todd Hewitt Broth and sub cultured on sheep blood agar and Chromogenic Strepto BID agar and incubated at 37으 for 24 hours. Identification was based on the Gram staining, presence of $\beta$-haemolysis and absence of catalase production. Antimicrobial susceptibility testing was performed using the Kirby-Bauer disk-diffusion methods. GBS colonization on average was confirmed in $27.8 \%$ of pregnant women and their neonate and proportion of GBS isolated from the vagina $6(30 \%)$ as compared to rectum $7(35 \%)$, neonates $4(20 \%)$, vaginal and neonate $1(5 \%)$, rectum and neonate $1(5 \%)$, and both vagina, rectum and neonate $1(5 \%)$. All isolates were found susceptible to $40 \%$ clindamycin $35 \%$ vancomycin, $90 \%$ ciprofloxacin $40 \%$ erythromycin and $100 \%$ resistance to penicillin used. There is need for proper handling of neonates by the health care practitioner and screening of pregnant women attending antenatal care, including known antibiotic Powered by Editorial Manager ${ }^{\circledR}$ and ProduXion Manager ${ }^{\circledR}$ from Aries Systems Corporation susceptibility for an appropriate antepartum antimicrobial prophylaxis can be offered.

Keywords: Antepartum; Neonate; Group B Streptococcus; Maternal morbidity; Postpartum; Antibiotics

\section{Omololu-Aso J*, Oluwatoyin Omololu-Aso O, Toluwalope Akinlolu J, Bukola Grace A and Micheal Tunde A}

Department of Microbiology, Obafemi
Awolowo University, Ile-Ife, Nigeria

*Corresponding author: Omololu-Aso J

omololu-aso@oauife.edu.ng

Department of Microbiology, Obafemi Awolowo University, Ile-Ife, Nigeria.

Tel: +2348033770933

Citation: Omololu-Aso J, Omololu-Aso OO, Akinlolu JT, Grace AB, Tunde AM (2017) A Case study of Group B Streptococcus Associated with Women in Antepartum Period and their Neonates, Ile Ife Southwestern Nigeria. Arch Clin Microbiol. Vol.8 No.6:68

Received: March 19, 2017; Accepted: November 06, 2017; Published: November 11, 2017

\section{Introduction}

Group B Streptococcus or Streptococcus agalactiae is one of the leading preventable causes of mortality and morbidity especially in neonate whose mothers are carrier worldwide [1]. Group B Streptococcus (GBS) is a Gram positive Bacterium regarded mainly as pathogen of pregnant or post-partum women and neonates [2].

It is also a significant cause of mortality and morbidity in non- pregnant adult, particularly those with underlying medical condition and in elderly patient such as stroke, renal failure, breast cancer, Diabetes mellitus, cirrhosis, neurological disorder and other liver disease, but with most incidence occurring among neonates [3].

Group B Streptococcus (GBS) is a normal flora or commensal of the genitourinary and gastrointestinal tract of $15 \%-50 \%$ of healthy woman [4]. Neonate can acquire GBS from their mother during the passage through the birth canal or through aspiration 
of infected amniotic fluid [5]. Vertical acquisition of GBS involving colonization of the skin or mucus membrane occurs in $15-50 \%$ new born to GBS colonized mother [6].

In Adult, Group B Streptococcus causes Urinary Tract Infection, osteomyelitis, pneumonia, meningitis, bacteremia, skin and soft tissue infection and Streptococcal Toxic Shock Syndrome [7]. The bacterium also causes high risk of preterm deliveries in pregnant woman [8]. Infections in new-borns occurring within the first week of life are designated Early-onset disease. Late-onset infections occur in infants aged $>1$ week, with most infections evident during the first 3 months of life (CDC, 2010) Figure 1.

GBS transmission rate from mothers to neonates through vaginal delivery is approximately $50 \%$, only $1-2 \%$ of colonized neonates develop invasive group B streptococcal disease [9]. The rate of early-onset infection has decreased from 1.7 cases per 1,000 live births in 1993 to 0.28 cases per 1,000 live births in the recent years (CDC, 2010). GBS colonization in women varies among age groups depending on study population, sites sampled and method of detection [10]. About $25 \%$ of pregnant women (CDC, 2010 ) and $35 \%$ young, non-pregnant woman [11] carry GBS in the rectum or vagina.

GBS continues to be susceptible to penicillin, ampicillin, and first-generation cephalosporins [12]. Under these circumstances, the alternative antibiotic choices have traditionally been erythromycin or clindamycin. However, resistance to these two antibiotics has been remarkably increasing [13]. Because of possible resistance problems with erythromycin and clindamycin, vancomycin is now the initial recommended treatment of GBS infection in patients who are allergic to penicillin [14].

Fluoroquinolones, especially the later generations, are active against GBS infections. Third and four-generation fluoroquinolones have been recommended by many health authorities and international organizations to treat pneumonia caused by GBS [15].

Maternal morbidity is worrisome, we source to determine the Social demographic factors with relative associated GBS colonization, its prevalence rate among pregnant women between 35-37 weeks of gestation period and their neonates, including the antibiotics susceptibility of the isolates recovered.

\section{fig 4.1: Antibiotic resistance profile of GBS} isolates

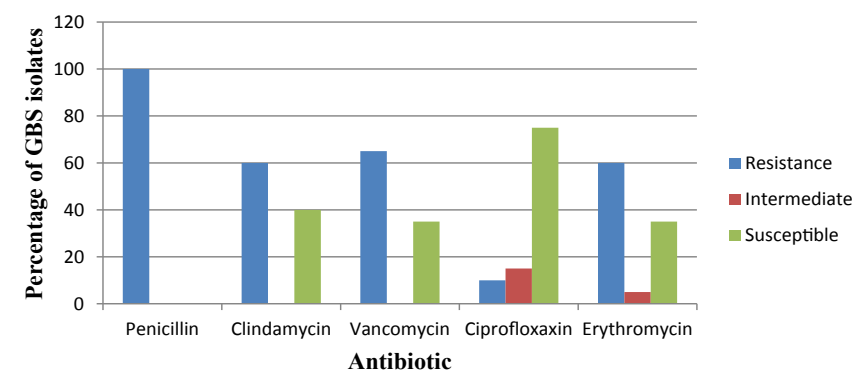

Figure 1 Antibiotics susceptibility profile of GBS isolates recovered in Ile Ife, South werstern Nigeria.
Streptococcus agalactiae was isolated from both the vaginal and rectum of pregnant women between 35-37 weeks of gestation and from the umbilical cord of their newly born neonates delivered at both labour ward and labour ward theatre between June 2016 to February 2017 at the Obafemi Awolowo University Teaching Hospital Complex (OAUTHC), Ile-Ife, Osun State, Nigeria.

\section{Sample Collection}

A total of 24 pregnant women samples were collected from each study site. Two sterile swab sticks were used to take two different samples from each patient. Vaginal sampling was carried out by inserting and rotating the sterile swab stick against the vaginal wall at the mid portion of the vault and the swab was carefully withdrawn to prevent contamination with the microflora of the vulva. Another sterile swab stick was inserted $1.5-2.0 \mathrm{~cm}$ beyond the anal sphincter and gently rotated to touch the anal crypts and finally sterile swab was used to swab the umbilical cord of the newly born immediately after delivery.

All samples were collected by Midwifery/Gynaecologist and the swab specimens were placed in Stuart transport media and were transported to Department of Microbiology Laboratory of OAU for Laboratory investigation.

\section{Screening for individual isolates}

After samples collection under aseptic conditions, the swab sticks were inoculated into selective enrichment broth medium (Todd-Hewitt broth supplemented with $10 \mu \mathrm{g} / \mathrm{ml}$ Colistinand 15 $\mu \mathrm{g} / \mathrm{ml}$ Nalidixicacid, Oxoid England) under aseptic condition and were incubated aerobically at $37^{\circ} \mathrm{C}$ for 24 hours. After 24 hours incubation, broth cultures were observed for growth (Turbidity) and then sub cultured onto 5\% sheep Blood Agar and incubated for $18-24$ hours at $37^{\circ} \mathrm{C}$ to increase the recovery rate of GBS. The plates that had no growth were re incubated overnight one more time. Where there were growth, the colonies were examined for their characteristics colonial morphology and beta-haemolysis and non beta-haemolysis were also considered. The suspected colonies were Gram stained (Gram positive) and tested for Catalase (catalase negative).

\section{Bacterial isolation and identification}

Rectum and vaginal swabs were collected from the third trimester pregnant women between 35-37 weeks of gestation and their neonate both at labour ward and labour ward theatre. The samples were cultured in Todd Hewitt Broth and sub cultured on sheep blood agar and Chromogenic Strepto B ID agar and incubated at $37^{\circ} \mathrm{C}$ for 24 hours. Identification was based on the Gram staining, presence of $\beta$-haemolysis and absence of catalase production. A known Staphylococcus aureus was used as positive control and Streptococcus pneumonia was used as a negative control.

\section{Antibiotic susceptibility test}

The GBS isolated sensitivity were tested with 5 different classes of antibiotic; vancomycin $(5 \mu \mathrm{g})$, penicillin $(10 \mu \mathrm{g})$, erythromycin $(15 \mu \mathrm{g})$ ciprofloxacin $(5 \mu \mathrm{g})$, clindamycin $(2 \mu \mathrm{g})$ Kirby-Bauer disc 
diffusion method was employed [12]. An inoculum suspension was prepared from a pure culture in a sterile nutrient broth using an inoculation loop. These were incubated overnight. The turbidity of the inoculums were adjusted and compared with 0.5 McFarland standards so as to obtain a confluent growth. Mueller-Hinton media plates were inoculated with a loop-full of the prepared inoculums and streaked by swabbing the surface of the medium rotating the plate through an angle of $60 \circ$ at each application. Finally, the swab was passed round the edge of the agar surface. The inoculated plates were left for a few minutes at room temperature with the lid closed. This allowed the moisture on the surface to be absorbed into the medium. The antibiotic discs were placed on the inoculated plates using a pair of sterile forceps. Each disc was gently pressed down to ensure even contact with the medium. A control was set up using $S$. pneumonia which was susceptible to all the antibiotics to be tested. The set up was incubated at $37^{\circ} \mathrm{C}$ for 24 hours in $5 \% \mathrm{CO}_{2}$. After overnight incubation, the diameter of each zone inhibition (including the diameter of the disc) of each antibiotic was measured and recorded in millimetres. The results were compared with the zone diameter interpretive standards of the Clinical Laboratory Standards Institute CLS.

\section{Results}

A total of 72 swabs were taken from 24 pregnant women at 35-37 weeks of gestation and their neonates at OAUTHC, 20 (27.8\%) were cultured positive for GBS based on Bergy's manual Biochemical test and systematic bacteriology. Morphological appearance of all the GBS positive isolates on chromogenic agar and the ability to lyse blood (from $5 \%$ sheep blood agar) were observed.

Most isolates were pink or cream in colour, circular in shape, there opacity is either opaque or translucent, entire in edges and have smooth surfaces. Catalase test were carried out on all the isolates to confirm that they were GBS, and all tested catalase negative. The isolates Gram stained and viewed under microscope using immersion oil, also all appeared purple cocci in chains. The isolates were identified based on Bergy's Manual of systematic Bacteriology.

\section{Discussion, Conclusion and Recommendation}

A total of 72 rectal and vaginal swabs were collected from 24 third trimester pregnant women and their neonates' umbilical cord. The result of the study showed higher prevalence rate of $27.8 \%$ Streptococcus agalactiae in this study area therefore confirming GBS colonization among pregnant women and the neonates. The findings agreed with studies of some workers in Tanzania whose reports shown colonization rates among mothers and neonates between $10 \%-36 \%$ [13]. Consequently, the result is higher than the prevalence rate as described in Nigeria by Onipede in Ile-Ife (11.3\%) [14] and Onile in Ibadan (Onile, 1980). The data however, are lower than data reported from Trinidad (32.9\%) and Zimbabwe $(31.6 \%)$ showing country variations [15]. Indicated variations could possibly due to differences in sampling techniques, study sites, populations investigated and the differences in type of culture media and culturing techniques used.

Table 1 summarized the association between GBS status and demographic characteristics of studied participant and the result revealed a higher colonization rate among age group $33-36$ years (13.89\%) which corroborated the report of Onipede where GBS was frequently isolated among older women with age $\geq 30$ years $(12.79 \%)$ against $(9.38 \%)$ in younger women [16]. These could be due to the fact that pregnant women with age range greater than 30 year were enrolled in the study. Colonization of GBS was higher in pregnant women with purulent vaginal secretion (18.06\%) as compare with pregnant women with mild secretion (9.72\%).

All isolates were found to be $100 \%$ resistant to penicillin this is similar to studies performed by onipede in Ile-Ife [17]. All isolates were also found Susceptible to $40 \%$ clindamycin, $35 \%$ vancomycin, $90 \%$ ciprofloxacin, 40\%erythromycin used. High level of resistance to Penicillin $G$ which is a first choice of drug for the treatment of GBS could be as result of drug abuse or

Table 1 Association between GBS status and Demographic Characteristics of Studied participants.

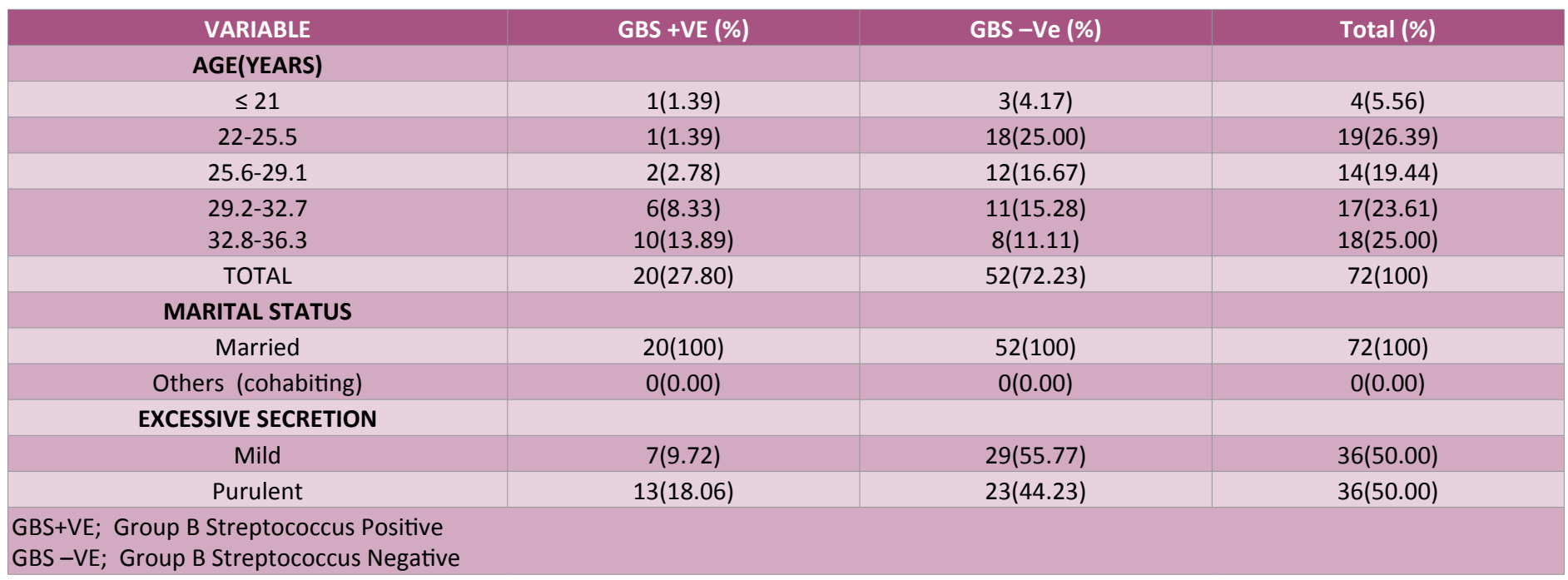


Table 2 Distribution Analysis for GBS-Infection.

\begin{tabular}{|c|c|c|c|c|}
\hline Sample Code & Trimester & PROM & $\begin{array}{l}\text { Antibiotic } \\
\text { Prophylaxis }\end{array}$ & $\begin{array}{l}\text { Catalase } \\
\text { reaction }\end{array}$ \\
\hline IV1 & $3^{\text {rd }}$ & NO & NO & - \\
\hline IB1 & $3^{\text {rd }}$ & NO & NO & - \\
\hline IR1 & $3^{\text {rd }}$ & NO & NO & - \\
\hline IV2 & $3^{\text {rd }}$ & NO & YES & - \\
\hline IB2 & $3^{\text {rd }}$ & NO & YES & - \\
\hline IV3 & $3^{\text {rd }}$ & NO & NO & - \\
\hline IR3 & $3^{\text {rd }}$ & NO & NO & - \\
\hline IB4 & $3^{\text {rd }}$ & NO & YES & - \\
\hline IR4 & $3^{\text {rd }}$ & NO & YES & - \\
\hline IB5 & $3^{\text {rd }}$ & NO & NO & - \\
\hline IB6 & $3^{\text {rd }}$ & NO & YES & - \\
\hline IB7 & $3^{\text {rd }}$ & NO & NO & - \\
\hline IB8 & $3^{\text {rd }}$ & NO & YES & - \\
\hline IV9 & $3^{\text {rd }}$ & NO & NO & - \\
\hline IV10 & $3^{\text {rd }}$ & NO & NO & - \\
\hline IV11 & $3^{\text {rd }}$ & NO & NO & - \\
\hline IR12 & $3^{\text {rd }}$ & NO & YES & - \\
\hline IR13 & $3^{\text {rd }}$ & NO & NO & - \\
\hline IR14 & $3^{\text {rd }}$ & NO & NO & - \\
\hline 1R15 & $3^{\text {rd }}$ & NO & NO & - \\
\hline
\end{tabular}

PROM; Premature/Preterm Rupturing of Membrane IV; Vaginal IR; Rectum IB; Baby

-ve; Catalase Negative+ve; Catalase Postive.

excessive use of the antibiotic in this part of the country and ease procurement of antibiotics in the country [18-23].

\section{Conclusion}

Certain degrees of prevalence of GBS infection among the pregnant women and neonates in this study area was observed and all the isolates recovered were found to be $100 \%$ resistant to penicillin happened to be the most widely used antibiotics for treatment of Group B Streptococcal infection. In this findings, ciprofloxacin remains the antibiotic of choice in treatment of Streptococcal infection.

Effective screening for GBS during antenatal care, Proper handling of neonates at postpartum by the health care practitioners with appropriate antepartum antimicrobial prophylaxis should be advocated.

\section{Recommendation}

- Women should be educated on GBS infection and the need to improve on personal hygiene.

\section{References}

1 Blasi FS, Ewig A, Torres G, Huchon (2006) A review of guidelines for antibacterial use in acute exacerbations of chronic bronchitis. Pulmonary Pharmacology Therapeutics 19: 361-369.

2 Brimil N, Barthell E, Heindrichs U, Kuhn M, Lutticken R, et al. (2006) Epidemiology of Streptococcus agalactiae colonization in Germany. International Journal of Medical Microbiology 296: 39-44.
- All pregnant women at 35-37 weeks of gestation should be screened for group B streptococcus Infection.

- Aseptic technique should be ensured in all prenatal caring process

- Since GBS has acquired resistance to several of the commonly used antibiotics, such as penicillin, prevention protocols other than antepartum chemoprophylaxis must be utilized to prevent neonatal disease.

\section{Study Design Limitation}

Based on our available procedures and constraint on study population at the centre, the findings may not be translated to be exact outcome of antepartum GBS prevalence rate associated with women in the country. However, the data obtained is of useful findings widely applicable as they will assist in control measures and effective therapeutic approach to GBS in pre-and post- natal stages among women in all part of the world.

Table 3 Multiple Antibiotic Resistannce Pattern of GBS Isolates.

\begin{tabular}{|c|c|c|c|}
\hline $\begin{array}{l}\text { Isolate } \\
\text { Code }\end{array}$ & Antibiotic Resisted & $\begin{array}{c}\text { Antibiotic } \\
\text { intermediate }\end{array}$ & $\begin{array}{l}\text { Antibiotics } \\
\text { susceptible }\end{array}$ \\
\hline IV1 & PEN, ERY & NONE & DA, VA, CIP \\
\hline IB1 & PEN, DA, VA & CIP & ERY \\
\hline IR1 & PEN, ERY & NONE & $D A, V A, C I P$ \\
\hline IV2 & PEN,VA,CIP & NONE & DA, ERY \\
\hline IB2 & PEN, VA & NONE & DA, CIP, ERY \\
\hline IV3 & PEN,DA, VA & NONE & CIP, ERY \\
\hline IR3 & PEN,DA, VA,ERY & NONE & CIP \\
\hline IB4 & PEN, DA, VA & NONE & CIP, ERY \\
\hline IR4 & PEN, VA & ERY & $\mathrm{DA}, \mathrm{CIP}$ \\
\hline IB5 & PEN, ERY & CIP & $\mathrm{DA}, \mathrm{VA}$ \\
\hline IB6 & PEN,DA, VA,CIP,ERY, & NONE & NONE \\
\hline IB7 & PEN, DA, VA & NONE & CIP,ERY \\
\hline IB8 & PEN, ERY & NONE & $\mathrm{DA}, \mathrm{VA}, \mathrm{CIP}$ \\
\hline IV9 & PEN, DA, VA & NONE & CIP, ERY \\
\hline IV10 & PEN, DA, ERY & CIP & VA \\
\hline IV11 & PEN,VA & NONE & DA,CIP, ERY \\
\hline IR12 & PEN,DA,ERY & NONE & VA, CIP \\
\hline IR13 & PEN, VA & NONE & DA,CIP, ERY \\
\hline IR14 & PEN,VA,DA, ERY & NONE & CIP \\
\hline IR15 & PEN, DA,ERY & NONE & VA, CIP \\
\hline
\end{tabular}

CIP: Ciprofloxacin VA Vancomycin DA: Clindamycin; PEN: Penicillin G E: erythromycin

3 Brochet M, Couve E, Bercion R, Sire J, Glaser P (2009) Population Structure of Human Isolates of Streptococcus agalactiae from Dakar and Bangui Journals of Clinical Microbiology 47: 800-803.

4 Castor ML, Whitney CG (2008) Antibiotic Resistance Patterns in Invasive Group B Streptococcal isolates. Infectious Disease Obstetrics Gynaecology 2008: 727505.

5. Centre for Disease Control and prevention (2010) Prevention of 
perinatal Group B Streptococcal disease-revised guidelines from Centre for Disease Control (CDC), 2010. Morbidity and Mortality Weekly Recommendations and Reports 59: 1-36.

6. Chen KT, Puopolo KM, Eichenwald EC, Onderdonk AB, Lieberman $E$ (2005) No increase in rates of early-onset neonatal sepsis by antibiotic-resistant group B Streptococcus in the era of antepartum antibiotic prophylaxis. American Journal Obstetric Gynecology 192: 1167-1171.

7. Edwards MS, Baker CJ (2000) Mandell, Douglas, and Bennett's Principles and practice of infectious Diseases. Churchil livingstone 21: 2156-2167.

8. Eren A, Kucukercan M, Oguzoglu N, Unal N, Karateke A (2005) The Carriage of Group B Streptococal in Turkish Pregnant Women and its Transmission Rate in New-borns and Serotype Distribution. The Turkish Journal of Paediatric 13: 28-33.

9. Gilbert DN, Moellering RC, Eliopoulos GM, Chambers HF, Saag MS (2011) The Sanford Guide to Antimicrobial Therapy. Antimicrobial activity therapy 12: 158 .

10. Huber CA, McOdimba F, Pflueger V, Daubenberger CA, Revathi G (2011) Characterisation of Invasive and Colonizing Isolates of Streptococcus agalactiae In East African Adults. Journal of Clinical Microbiology1: 1288-1311.

11. Janapatla RP, Ho YR, Yan JJ, Wu HM, Wu JJ (2008) The Prevalence of Erythromycin Resistance in Group B streptococcallsolates at a University Hospital in Taiwan. Microbial Drug Resistance14: 293-297.

12. Joachim A, Matee MI, Massawe FA, Lyamuya EF (2009) Maternal and Neonatal Colonisation of Group B Streptococcus at Muhimbili National Hospital in Dar es Salaam, Tanzania: Prevalence, Risk Factors and Antimicrobial Resistance BMC Public Health 9: 437.

13. Madzivhandila M, Adrian PV, Cutland CL, Kuwanda L, Schrag SJ, et al. (2011) Serotype Distribution and Invasive Potential of Group B Streptococcus Isolates Causing Disease in Infants and Colonizing Maternal-New-born Dyads. PLoS One 6: 17861.
14. Manning SD, Foxman B, Pierson CL, Tallman P, Baker CJ, et al. (2003) Correlates of Antibiotic-Resistant Group B Streptococcus Isolated from Pregnant Women. Obstetrics Gynecology101: 74-79.

15. Najmi N, Sikandar R, Zuberi NF, Jehan I (2013) Maternal Genital Tract Colonization by Group B Stretococcus: A Hospital Based Study. Journal of Pakistan Medical Association 63: 1103-1107.

16. Nsagha DS, Bello CSS, Kandakai-Olukemi YT (2012) Epidemiological significance of the colonization of Streptococcus agalactiae in the anorectum and endocervix of non-patrturients in Jos Nigeria. African journal of clinical and experimental microbiology13:144-149.

17. Okeke IN, Lamikanra A, Edelman R (1999) Socioeconomic and Behavioral Factors Leading to Acquired Bacterial Resistance to Antibiotics in Developing Countries. Emerging Infectious Disease 5: 18-27.

18. Onile BA (1980) Group B Streptococcus Carriage in Nigeria. Transaction Royal Society of Tropical Medicine and Hygiene 74: 367-370.

19. Onipede A, Adefusi O, Adeyemi A (2010) Group B Streptococcus Carriage during Late Pregnancy in Ile-Ife, Nigeria. African Journal of Clinical and Experimental Biology 13: 135-143.

20. Oviedo P, Pegels E, Laczeski M, Quiroga M, Vergara M (2012) Phenotypic and genotypic characterization of Streptococcus agalactiae in pregnant women. First study in a province of Argentina. Brazilian Journal of Microbiology 44: 253-258.

21. Panda B, Iruretagoyena I, Stiller R , Panda A (2009) Antibiotic Resistance and Penicillin Tolerance in Ano-Vaginal Group B Streptococci. Journal of Maternal Fetal Neonatal Medicine 22: 111-114.

22. Skoff TH, Farley S, Petit AS, Craig W, Schaffner K (2009) Increasing Burden of Invasive Group B Streptococcal Disease in Nonpregnant Adults, 1990-2007. Clinical Infectious Disease 49: 85-92.

24. Wang YH, Su LH, Hou JN, Yang TH, Lin TY, et al. (2010) Group B Streptococcal Disease in Non-Pregnant Patients: Emergence of Highly Resistant Strains of Serotype Ib. in Taiwan, 2006-2008. Journal of Clinical Microbiology 8: 8-10. 